\title{
In Defense of the Metaphysics of Entanglement ${ }^{1}$
}

David Glick $^{2}$ and George Darby ${ }^{3}$

\begin{abstract}
Quantum entanglement has long been thought to be have deep metaphysical consequences. For example, it has been claimed to show that Humean supervenience is false or to involve a novel form of ontological holism. One way to avoid confronting the metaphysical consequences is to adopt some form of antirealism. In this paper we discuss two prominent strands in recent literature-wavefunction realism and "Super-Humeanism"- that appear quite different, but, as we see it, are instances of a more general strategy. In effect, what these attempt to do is to diffuse the puzzle of entanglement by eliminating it. These interpretative movements are advertised as equally realist, but, we claim, fail to take an appropriately realist attitude towards entanglement. What we advocate instead is a genuine metaphysics of entanglement: instead of eliminating entanglement, develop a metaphysics that accounts for and explains it.
\end{abstract}

\section{Introduction}

Anyone who accepts quantum theory must allow that entanglement exists, in some sense. But it's important to distinguish several related claims:

1. Non-locality: The existence of certain non-local phenomena, such as measurement results in an EPR experiment.

2. Non-separability: The existence of composite systems assigned quantum states that cannot be recovered from the quantum states of the constituents of the system.

3. Entanglement realism: The existence of some entity or entities in our fundamental ontology constitutive of entanglement.

The first two theses are relatively uncontroversial, but as we'll see, the lattermost is a point of contention among interpreters of quantum theory. Our focus here will be with (3), but let's briefly consider (1) and (2) before setting them aside.

\subsection{Non-locality}

Quantum theory predicts correlations in measurement outcomes incapable of locally causal

\footnotetext{
${ }^{1}$ Forthcoming in Glick, Darby, Marmodoro (eds.) The foundation of reality: Fundamentality, space, and time. OUP.

${ }^{2}$ University of Sydney | david.glick@sydney.edu.au

${ }^{3}$ University of Durham | george.a.darby@googlemail.com
} 
explanation. This follows from Bell's theorem, which shows that any locally causal theory must conform to Bell's inequality, which is violated by the predictions of quantum theory. After the experiments of Aspect in 1982 and those following him, we may now say that "[V]iolations of Bell's inequality show that the world is nonlocal" (Maudlin 2011, p.111, original emphasis).

Non-locality, then, is hard to deny. It's an observable fact about our world that such correlations exist. Even those views that deny the reality or fundamentality of measurement outcomes must accept non-locality in some form at some level of description. On the Everett interpretation, for instance, the experience of an agent will almost certainly include evidence of non-local correlations in measurement outcomes. Non-locality is part of our evidence for quantum theory, and hence, it must be recovered by any interpretation lest it be empirically incoherent (Barrett 1999). 4

\subsection{Non-separability}

The term "non-separable" is sometimes taken to have metaphysical implications. Our use here has no such implications. The claim is simply that the application of quantum theory involves assigning quantum states to composite systems that cannot be factored into quantum states of their constituents. It is a further claim---which some deny---that these states represent particular physical properties or entities.

The standard example of non-separability is a two-particle system assigned a pure state $\Psi_{12}$ that doesn't factor into pure states of the individual particles: $\Psi_{12} \neq \Psi_{1} \otimes \Psi_{2}$ where $\Psi_{12} \in H_{12}, \Psi_{1} \in$ $H_{1}, \Psi_{2} \in H_{2}$ and $H_{12}=H_{1} \otimes H_{2}$. One can also define non-separability for an arbitrary quantum state in terms of density operators. Here the quantum state of the joint system, $\rho_{12}$, is non-separable just in case it violates: $\rho_{12}=\sum_{i} \alpha_{i} \rho_{1}{ }^{i} \otimes \rho_{2}{ }^{i}$, with $\alpha_{i}>0, \sum_{i} \alpha_{i}=1$. Non-separability is a widely accepted feature of the standard quantum formalism and its application. There are difficult questions about the correct formal structure for quantum theory in general, and how to

\footnotetext{
${ }^{4}$ Wallace (2012) claims that Bell's theorem simply doesn't apply to the Everett interpretation given the latter's denial of unique measurement outcomes. And non-ontic view such as QBism and Healey's pragmatism claim to be fully local. However, the point remains that when Alice and Bob compare measurement results from an EPR experiment they (almost certainly) find violations of Bell inequalities. One may deny the inference from these correlations in reports to correlations in reality, but even so, there will remain an analog to non-locality in reports---once Alice finds out Bob's measurement results, she finds violations of Bell inequalities. Denying the veridicality of such reports brings one perilously close to solipsism (Norsen 2016).
} 
characterize non-separability in particular, but it remains a core feature of any quantum theory that some states will have this character. Indeed, in introducing entanglement, Schrödinger (1935) called non-separability "not one but rather the characteristic trait of quantum mechanics" (p.555, original emphasis). Thus, any view that accepts the quantum formalism in anything like its standard form will allow for the non-separability of quantum states.

\subsection{Entanglement realism}

Entanglement realism requires more than merely recognizing the existence of non-local phenomena and non-separable quantum states. In particular, it requires that non-separable states represent genuine entanglement in the world. The details of how entanglement is understood may vary, but the realist regards entanglement as part of the structure of our world rather than a mere appearance. Consider an analogy with retrograde motion, the apparent "backwards" motion of certain planets through the sky relative to the background of stars. On the Ptolemaic astronomical model, planets travel along circular orbits around the earth and smaller epicycles around a point on the main orbit. The combination of the motion along the epicycle and the main orbit allows a planet to travel backwards for brief periods of time, allowing for their retrograde motion. On the Copernican model, however, planets travel along orbits around the sun and apparent retrograde motion occurs when a planet overtakes, or is overtaken by, the earth. Hence, retrograde motion is real on the Ptolemaic view, but merely apparent for the Copernican. It seems natural to say that the Ptolemaic position is realist about retrograde motion while the Copernican position is antirealist.

Analogously, the entanglement realist embraces entanglement as a genuine feature of reality that is not merely apparent. This licenses the metaphysics of entanglement, which seeks to understand the nature of this feature of our world. Although the term may not be in wide usage, one can find several authors gesturing at entanglement realism. For instance, Wallace (2012), in describing entanglement on the Everett interpretation says that "picturesquely, we can think of entanglement between states as a string connecting those states, representing the nonlocal relation between them" (p.304). Healey (2012) rejects entanglement realism when he argues against the claim that "ascribing an entangled state to quantum systems is a way of representing some new, non-classical, physical relation between them" (p.31). And Esfeld (2004) suggests a version of entanglement realism when he claims that "[w]hatever entanglement may exactly be, it is a relation among quantum systems. 'Being entangled with' is 
a property that is predicated of at least two quantum systems; it is thus a relational property" (p.604).

There are several ways of denying entanglement realism. Most straightforwardly, views that deny the quantum state a representational role do not find entanglement in the world. QBism, for instance, takes quantum states to encode an agent's beliefs as they relate to certain experimental procedures and future experiences (Fuchs et al. 2014). Healey's pragmatist view of quantum theory differs significantly from QBism, and gives quantum states a more objective status (Healey 2017). But, Healey agrees that the primary function of an entangled state is to tell an agent to expect Bell-type correlations when comparing the outcomes of certain experiments. On such views, entanglement, as a relation between quantum states, concerns a particular kind of belief an agent might have-one which would lead them to expect non-local phenomena. $^{5}$

Such non-representational views aren't the only way to be an antirealist about entanglement. An interpretation may grant the quantum state a representational role, but still deny that entangled states describe worldly entanglement. Indeed, below we claim that two "realist" approaches to the metaphysics of quantum theory are committed to entanglement antirealism. On these views, quantum states do sometimes describe features of the world, but not in a manner that countenances entities, properties, or relations constitutive of entanglement. Wavefunction realism and Super-Humeanism (or "Bohumeanism") are often motivated by a desire to avoid what are perceived as problematic metaphysical implications of entanglement, but we argue that these problems aren't so great as to motivate eliminating entanglement in the manner they suggest.

Before closing this introduction, it's worth noting that, other things being equal, realism has certain benefits. First, it accords with a flat-footed understanding of the metaphysical implications of physics. While it's clear that one cannot read-off metaphysics from physics, it is nevertheless an aim of naturalistic metaphysics to let physics guide much of our metaphysical theorizing. The realist attitude recommends taking theories at face-value and committing to the ontology they describe. ${ }^{6}$ Second, realism has certain explanatory power. The realist is able to

\footnotetext{
${ }^{5}$ Or reports of apparently non-local phenomena. See footnote 1.

${ }^{6}$ Of course, it's sometimes impossible to take a theory at face-value, and hence some amount of
} 
provide explanations involving the nature of reality itself rather than merely being limited to the predictive features of our scientific theories. In the case of entanglement, this suggests that the realist will be able to offer explanations of non-local phenomena unavailable to the anti-realist---viz. those based in genuine physical entanglement. ${ }^{7}$

\section{Humeanism and entanglement}

One motivation for abandoning realism about entanglement is the perceived threat to the doctrine of Humean Supervenience, which lies at the heart of Lewis's neo-Humean approach to metaphysics. Humean Supervenience, in Lewis (1986), says that everything supervenes on the monadic properties of point-like things plus spatiotemporal relations. But entanglement seems to show that there are things that don't supervene on that basis, and hence, that Humean Supervenience is false.

The response that we favor is that this problem for Humean Supervenience is nowhere near as serious as is often made out. This is because, for all that has been shown so far, it is perfectly compatible with Humean Supervenience to simply include in the supervenience basis whatever troublesome relations are supposed to be required by entanglement. Obviously one cannot do that for every threat to Humean Supervenience, or the thesis would be trivial. But so long as the relations to be added are not powers, or lawmaking relations between universals, or otherwise obviously unHumean, it seems that admitting, say, an "opposite-spin" relation into the ontology will do no harm at all - the natural thought would be that such relations can be analyzed as "external" in Lewis's sense. And, surely, the burden of proof is at least partly on the anti-Humeans to show that the relations in question cannot be thus analyzed-and nothing like that has been shown so far. Since the context of much of this discussion is in the movement towards "naturalistic" metaphysics - the demand for metaphysicians to take notice of natural science-the proper response for Humeans is to accept the extra ontology and await a demonstration that it conflicts with their ideology.

An alternative response, which we follow Esfeld and Deckert (2017) in calling

(re-)interpretation is required. The point here is that realism requires an attitude or methodology of beginning from a flat-footed literal reading of a physical theory and going from there.

${ }^{7}$ We will return to explanatory power of entanglement realism in section 3 below. 
"Super-Humeanism"-though as will become apparent it is not clear that there is anything super about it-is advocated in various forms by Esfeld and Deckert, Miller (2014) (under the heading "Bohumeanism"), and Bhogal and Perry (2017). Our claim in this section is that Humeans should reject Super-Humeanism and should take the more neutral stance, holding that whatever the fundamental ontology turns out to be, it will be analyzable in terms of some fundamental things standing in natural external relations.

The reasons for this are best seen in light of the alleged advantages of Super-Humeanism; we will focus on two: 1 . The claim that Super-Humeanism is somehow more faithful to the true nature of entanglement, and 2. The claim that it avoids quidditism. We'll argue that both claims are problematic.

\subsection{Super-Humeanism and the nature of entanglement}

Under the heading of "Quiddistic Entanglement," Bhogal and Perry consider something like the Humean response that we prefer. They say that a way out of the conflict between Humean Supervenience and QM:

"...is to deny that entangled states imply anything about any other states. This view accepts that there is a world where two particles are in a Singlet state but yield matching outcomes to properly calibrated $x$-spin measurements. This is an option for the Humean. It's major drawback is that it involves a unintuitively quiddistic conception of entanglement-it violates the intuition that entanglement implies something substantive about the particles so entangled. Perhaps such a quidditism isn't so bad for the Humean, after all if the Humean is to use recombination she will require a quiddistic conception of the properties that make up the mosaic. But our view does not have a quiddistic conception of entanglement; it retains a more intuitive understanding of the phenomena." (Bhogal and Perry 2017, p.8)

The option rejected here seems to us to be exactly the right one to consider: Humeans are already committed to properties understood as quiddities, so why not have a few more? This parallels the preferred response above: Lewis is already committed to a class of natural external relations, so why not accept some more to account for entanglement. We accept, of course, that entangled states imply something about their substates; what we deny is that they imply 
anything with metaphysical necessity. The intuition that entanglement implies something substantive about the particles so entangled is satisfied by their instantiating natural external relations beyond the spatiotemporal. Anything more involved than that is unHumean.

The claim that entanglement "implies something substantive about the particles so entangled" may mean a number of things, of course. Take a view on which the entangled particles somehow cease to become numerically distinct, or are "non-individuals," or something similar. This kind of view does indeed appear to imply something very substantive about the particles. Or suppose that one thought that the correspondence between the part-whole relation and a relation of ontological dependence somehow became inverted in entanglement: whereas usually the whole depends on the parts, now the parts depend on the whole; one can see how entanglement would then imply something strikingly substantive about the particles. ${ }^{8}$ In a sense, we want to say that the entanglement-as-external-relations view also implies something substantive about the entangled particles of our world-viz. that they instantiate a certain natural relation. On the other hand, there is nothing implied about the particles considered individually-indeed, that is the whole point.

The Super-Humean view also, of course, implies nothing substantive about the particles considered individually-in some variants they are just featureless blobs. Nor is there anything even as substantive as the instantiation of a natural relation-the whole point of a Humean treatment is, of course, to make things unsubstantive-Humeans being suspicious of substantive things like powers and necessary connections as being ultimately unintelligible. ${ }^{9}$ So, in a sense, the Super-Humean view can't imply anything substantive about entanglement, because Super-Humeanism doesn't imply anything substantive about anything. What the Super-Humean view does imply, and presumably the advantage that Bhogal and Perry have in mind, is that there is something about entangled particles that is necessarily true about entangled particles: necessarily, if $x$ and $y$ are entangled then $p$, where $p$ is a fact involving laws and thus ultimately-on the best-system view of laws-about the whole mosaic. But what is unclear now is why Humeans should particularly value that.

\footnotetext{
${ }^{8}$ See section 3 below for a discussion of this approach to the metaphysics of entanglement.

${ }^{9}$ But again, the point of the entanglement-as-external-relations view is that Humeans already accept some natural relations, so why not a few more?
} 
Maybe we should understand "substantive" as something like observable. Bhogal and Perry need not mean actually observable, but perhaps reflected somehow or other in the pattern exhibited by the Humean mosaic. And it does do this, of course: to be entangled is just a matter of things being thus and so in the Humean mosaic. So obviously being entangled implies—strictly implies—something about the mosaic. ${ }^{10}$

But, as Bhogal and Perry acknowledge, Humeans, especially those following Lewis, are already in the business of denying that many things hold of metaphysical necessity. In a different context, but illustrating perfectly the general attitude, it famously seems that on Lewis's theory of transworld identity, Lewis should happily accept that he could have been a fried egg. Nothing particularly substantive, in this sense, is implied about anything, and it is unclear why entanglement should be an exception.

\subsection{Humeanism and quidditism}

Esfeld and Deckert, and Bhogal and Perry, all suggest that avoiding quidditism is a desirable feature of the Super-Humean strategy over regular Humeanism. But is it really?

The first question is what exactly is supposed to be wrong with quidditism anyway. Esfeld and Deckert (2017) say that Lewis's quidditism is "occult" (p.46) and "baroque" (p.49). But it's not like quidditism is an extra bit of ideology, like powers again, it's just a thesis about the distribution of stuff over the plurality or about the extent of the plurality. It's hard to see what exactly is "baroque" about that. The question of whether quidditism is occult raises an interesting parallel with what exactly Humeanism is supposed to be, and why we should try to defend it in the apparent conflict with quantum mechanics.

One way of understanding Humeanism is available once one buys into the rest of the Lewisian ontology: Once we have laid out before us the whole of the Lewisian plurality of worlds, we simply ask: is it the case that $A$ and $B$ are in one world, but in no world is there $A$ but not $B$ ? If $A$ and $B$ qualify as "distinct existences", then this would be a "necessary connection between

\footnotetext{
${ }^{10}$ It implies something either directly, in terms of the outcomes of measurements actually performed, or indirectly, in terms of the outcomes of measurements that are not, but could be, performed-such counterfactual outcomes also being a matter of the mosaic (everything supervenes on the mosaic, including counterfactuals).
} 
distinct existences": claims of necessary connection are just modal claims, and those are evaluated in the usual way.

Quidditism, in the Lewisian idiom (e.g., modality is captured by bog-standard first order quantification), concerns what Esfeld and Deckert call indiscernible worlds. In some contexts, two worlds are indiscernible if they have exactly the same Humean mosaic-i.e., if the pattern of instantiation of fundamental properties and relations (and thus also the laws, since the laws are determined by the pattern of instantiation of fundamental properties and relations), are the same. In Esfeld and Deckert's usage, two worlds are indiscernible if, so far as their inhabitants could tell, the pattern of instantiation of fundamental properties and relations (and thus also the laws), are the same. Since epistemic access to the fundamental properties and relations is via discovery of the laws, the inhabitants of such worlds ( $w$ and $\left.w^{*}\right)$ cannot tell which of those two worlds they are in.

Just as haecceitism can be thought of as the metaphysical apparatus ("haecceity", "primitive thisness", etc.) that allows the circumstance that an individual may occupy some role in one world but not in another, even though it has the relevant properties in both, so quidditism can be seen as the metaphysical apparatus that allows the circumstance above (viz. that there are worlds $w$ and $w^{*}$ that are indiscernible in the second sense). But-especially in the case of haecceitism, the metaphysical apparatus in question is extremely murky, and so it is better to think directly about the circumstance in question-thus, we can simply ask whether or not there are haecceitistic differences-and the question of whether there are haecceitistic differences is simply a question about the way in which the Lewisian plurality is. In the case of quidditism, the background metaphysics is not quite so murky-if universals are what make for natural properties then they seem to deliver quiddities easily enough-but again it is cleanest to talk simply in terms of quidditistic differences. So, our metaphysics is committed to quidditism if there are worlds like $w$ and $w^{*}$ above.

So, then, the question is just whether there are worlds like $w$ and $w^{*}$, and given Lewis's recombination principle the answer seems to be obviously yes. So, quidditism follows. On this way of thinking, quidditism is just a claim about what worlds there are. It's no more or less occult than modal realism. 
There is a different way of thinking about what Humeanism is: the rejection of "occult" metaphysics such as powers. In a similar way, one can think of quidditism not in terms of the way the plurality is, but in terms of the way in which Lewis understands natural properties: are there universals, for example, to correspond to natural properties? If so, then quidditism again easily follows, because properties just correspond to universals, so of course universal $F$ can play the same role in one world as universal $G$ plays in another-this just requires the right pattern of instantiation of $F$ and $G$. But again, there is nothing "occult" about this-it's just the way of thinking about natural properties that is central to Lewis's metaphysics, and is only occult if natural properties are (Lewis 2009, p.210).

Esfeld and Deckert advertise the avoidance of quidditism as an advantage of Super-Humeanism because (a) quidditism, and quidditistic humility, are inessential features of true Humeanism, and, moreover, are undesirable features, and (b) their metaphysics avoids quidditism but is in other respects like Lewis's Humeanism. But for the reasons above, (a) is unconvincing. As for (b), does the Super-Humean strategy really avoid quidditism? In a sense, of course, they have avoided quidditism for monadic properties by not having any monadic properties. But, they do still have relations-the fundamental distance relations-so why are they not still committed to quidditism about the relations? And if they are, then doesn't this undermine the whole argument? Quidditism about relations is still quidditism!

Now of course there may be reasons why entanglement relations must be different in kind from spatiotemporal relations, such that one could see spatiotemporal relations-but not entanglement-in a non-quidditistic way, but that has to be spelled out, and that has not been done. Moreover, it seems clear that we do have quidditism about the distance relations:

In the discussion of quidditism, and consequent humility about the fundamental properties, Lewis (2009) proposes to "speak of 'fundamental properties' for short, but they fall into several categories. There are all-or-nothing monadic properties. There are all-or-nothing $n$-adic relations, at least for smallish $n$. There are properties that admit of degree" (p.205). Spatiotemporal relations appear to admit of degree, so cannot perhaps be thought of as all-or nothing; but what seems certain is that so far as quidditism and humility are concerned, what goes for monadic properties goes too for relations. Thus, in discussing the consequences of quidditism, Lewis takes for granted that humility extends to relations. ${ }^{11}$

11 "Suppose we have a structural property: say, the property of being composed of an F bearing relation $\mathrm{R}$ 
Thus, it seems clear that quidditism applies to relations just as much as to monadic properties. And so nothing is gained, from this point of view, by moving to an ontology that includes only relations among the fundamental properties. Thus there is nothing super about "Super-Humeanism" over bog-standard Humean supervenience; apart, of course, from the fact that Super-Humeanism is apparently compatible with quantum mechanics whereas standard Humean superveience is not. But then, of course, the obvious move of acknowledging the lessons of entanglement rather than evading them, and making the appropriate additions to the supervenience basis, works just as well to achieve the required reconciliation.

\section{Holism and entanglement}

Another strand of entanglement antirealism is motivated by the suggestion that entanglement involves a kind of ontological holism. Some forms of holism clearly endorse entanglement realism. For instance, the relational holism of Teller (1986) maintains that entanglement is manifested in relations between entangled particles that fail to supervene on their intrinsic properties. Relational holism is closely-aligned with the approach we favor in the Humean context, and shares many of its virtues. ${ }^{12}$ However, other forms of holism have the effect of eliminating entanglement from fundamental reality. In particular, wavefunction realism maintains that the fundamental ontology of physical reality is located in a high-dimensional space free from non-local influences and entanglement relations.

"Wavefunction realism" is a misleading term. A number of views claim that the wavefunction (or quantum state) is real and reserve a place for it in their fundamental ontology. The version of wavefunction realism we have in mind here, is the one defended in, e.g., Albert (1996, 2013); Loewer (1996); Ney (2012, 2013, 2015); North (2013). On this view, the wavefunction is understood as a physical field defined on a high-dimensional space-in the case of ordinary non-relativistic quantum mechanics, a $3 n$-dimensional configuration space,

to a $G$, where $F, R$, and $G$ are fundamental. If we do not know the identities of the properties we have called ' $F$ ', 'R', and ' $G$ ' we also do not know the identity of the structural property constructed out of them" (Lewis 2009, p.215).

12 Unlike the Humean approach discussed above, Teller maintains that spacetime is substance, and hence, that spatiotemporal relations do supervene on the intrinsic properties of their relata (i.e., are internal rather than external). Thus, he takes the relational holism implied by entanglement to be a novel feature of the quantum world. 
where $n$ is the number of particles that exist. Because the wavefunction is part of the most fundamental description of the world, and is defined on configuration space, it is this $3 n$-dimensional space rather than ordinary 3-dimensional space that is fundamental. Far from denying the reality of entanglement, defenders of wavefunction realism often point to entanglement and non-locality as sources of motivation.

There are at least two arguments used to motivate wavefunction realism on the basis of entanglement. The first is formal in nature. It begins with the observation that one cannot adequately characterize an entangled system with wavefunctions defined on 3-dimensional space. One cannot distinguish an entangled pure state from a similar mixed state using only individual position wavefunctions in 3-space. "To capture the difference between [pure and mixed] states, we must use the additional spatial degrees of freedom we have in configuration space. Entanglement is a completely pervasive feature of a quantum world, and what is needed to capture states that are entanglements of position... is a configuration space representation" (Ney 2013, p.17). This point is correct as far as it goes, however, the fact that individual position wavefunctions in 3-space are incapable of capturing entanglement needn't recommend moving to configuration space. One may opt instead to (a) adopt a different understanding of the quantum state than one based on individual position wavefunctions (e.g., the spacetime state realism of Wallace and Timpson 2010) or (b) recognize more than individual position wavefunctions in one's fundamental ontology (e.g., physical relations constitutive of entanglement). Thus, this motivation for moving to configuration space will only appeal to those who already accept a fundamental ontology free from entanglement.

Another argument from entanglement to wavefunction realism is explanatory in nature. In light of Bell's theorem, one cannot explain non-locality in familiar causal terms without invoking action at a distance. ${ }^{13}$ However, Ismael and Schaffer (2016) argue that a non-causal explanation of non-locality can be given in terms of "common ground." The core idea is that if entangled particles are fundamentally non-distinct, then correlations in measurement outcomes involving them may be accounted for by appeal to grounding relations-properties of the individual particles are determined by properties of their common ground. There are a variety of

\footnotetext{
${ }^{13}$ We are taking "familiar causal terms" here to refer to either a direct causal connection or a common cause explanation capable of "screening-off" the correlata in the sense of Reichenbach (1971).

Retrocausal explanations are also being set aside.
} 
ways of developing a common ground account, some of which are eliminative and some of which aren't. Our contention here is that the non-eliminative versions do a better job of explaining what's special about entanglement.

Wavefunction realism may be considered to be a version of the common ground approach. For the advocate of wavefunction realism, the manifestation of non-locality in 3-space is a reflection of a more fundamental state of affairs in 3n-space. In particular, the coordinated behavior of distinct particles in 3-space is explained by appeal to the fact that, fundamentally, they have a common ontological basis in the fundamental $3 n$-space. Moreover, the fundamental states of affairs involve no non-locality; happenings at one location in $3 n$-space are independent of happenings at "distant" locations. ${ }^{14}$ Thus, wavefunction realism seems to offer the prospect of a satisfying ontic explanation of non-locality that avoids action at a distance, or fundamental entanglement in the world.

The problem is that this implementation of the common ground strategy is global insofar as everything in 3-space has a common basis in the ontology of $3 n$-space. The particles involved in an EPR experiment are fundamentally non-distinct, for example, but so are any pair of particles anywhere in the world. Arguably, this results in a kind of (priority) monism, as defended by Schaffer (2010). But part of what is required in an explanation of non-locality is an account of what is special about the systems that manifest it, and on monism, having a common ground is a perfectly generic feature of everything found in 3-space.

Schaffer (2010) argues that entanglement ("non-separability" in our terminology) is itself perfectly generic in quantum theory, and hence, that monism is well supported. This suggests that wavefunction realism may provide a satisfying common ground explanation after all. However, there are several issues with this reply. First, it's not at all clear that "the cosmos forms one vast entangled system" (ibid., p.52). While it is true that some interpretations of quantum theory may recognize a universal wavefunction in which the entire universe is described by a massively-entangled quantum state, other interpretations reject either: (a) that there is a universal wavefunction or (b) that such a state would involve widespread

\footnotetext{
${ }^{14}$ Note that the "locality" of points in configuration space is only analogous to locality in ordinary space. The notion of "distance" involved is defined by a metric on $3 n$-space, analogous but not identical to the familiar notion of spatial distance.
} 
entanglement. Second, even if there is an entangled universal wavefunction, there is a distinction to be drawn between subsystems that display non-local behavior and those that don't. Careful preparation is required to display non-local phenomena capable of violating a Bell inequality, and the vast majority of observable phenomena do not display non-locality in this sense. Believers in an entangled universal wavefunction typically appeal to decoherence to establish the required distinction (e.g., Wallace 2012), but then it seems to be decoherence rather than common ground doing the explanatory work. In sum, an appeal to common ground cannot explain non-locality in the sense of saying what's special about systems that display it if every physical system shares in that common ground.

According to the wavefunction realist, the appearance of entanglement in 3-space is evidence that there is something wrong in our choice of fundamental space. They advocate moving to $3 n$-space where problematic non-locality is no longer present. In moving to a higher-dimensional space, the wavefunction realist has eliminated entanglement rather than embraced it. The wavefunction realist advertises this as an explanation of entanglement, but it more resembles an error theory. It can explain why there appears to be entanglement, but in the end, it is only a feature of the redundant representation of a reality free from entanglement (Ismael, forthcoming). Thus, for the wavefunction realist, entanglement is not a part of the fundamental structure of the world.

Fortunately, one can take up the common ground strategy without eliminating entanglement. The key is to locate entanglement in the same space where we find non-locality, that is, ordinary 3-space. ${ }^{15}$ The relations of ontological dependence (or grounding) that link entangled physical systems can obtain without invoking a more fundamental non-spatiotemporal level of reality. For example, consider a case of ordinary physical composition, say, hydrogen and oxygen atoms composing a water molecule. One may regard the composite systems-the water molecule-as dependent on (or grounded in) the atoms that compose it. As a result, features of the composite may be explained by features of its constituent parts. For instance, the overall electrical neutrality of the (non-ionized, liquid state) water molecule may be explained by the respective charges of the hydrogen and oxygen atoms (ions). It is often supposed that ontic explanations

\footnotetext{
${ }^{15}$ Or, more accurately, 4-dimensional spacetime. We will continue to speak in terms of $3+1$ dimensional space and time as this is the setting in which ordinary, non-relativistic quantum mechanics is often situated. That said, none of the arguments given depend on this being the fundamental space rather than relativistic spacetime.
} 
require relations of dependence (causal or non-causal), so that there must be a real-world dependence relation underlying explanations like this one (Koslicki 2012, p.213; Schaffer 2017, pp. 308-9). In this case, the dependence relation is that of a whole on its parts. But, given that ontological dependence and composition are distinct notions, it's at least conceptually possible that parts could depend on wholes.

Thus, one can maintain that entanglement involves cases of part-on-whole dependence. Two electrons in the spin singlet state depend on the two-electron whole they compose. This dependence relation, in turn, can support an ontic explanation of the anti-correlation of their spins described by quantum theory: the two-electron system has net spin $=0$, hence, if one electron is spin $+1 / 2$ along a given axis, the other electron must be spin $-1 / 2$ along that axis. This has the same structure as the ordinary case, only with the direction of part-whole dependence reversed. Unlike wavefunction realism, this version of the common ground story needn't be global; part-on-whole dependence is a unique feature possessed by all and only entangled systems. Hence, the account recognizes entanglement in the form of characteristic relations of ontological dependence in ordinary 3-space.

This style of explanation also admits of an alternative reading. It could be that part-on-whole dependence indicates we have individuated systems in too fine-grained a manner. For instance, if we try to treat a system as having parts that it lacks, then we should expect to find this non-standard pattern of ontological dependence. Thus, when we attempt to ascribe quantum states to entangled particles-e.g., by taking the partial trace of the density operator for the composite system-we are carving up reality into smaller bits than it really contains. On this reading, entanglement doesn't consist in a unique form of ontological dependence, but rather the ontological holism of entangled composites (Healey 2016). This brings us close to the motivation for wavefunction realism discussed above, but there remain two crucial differences: (a) the wholes in question are located in 3-dimensional space, and (b) all and only systems of mutually entangled particles are wholes. Thus, only if everything is truly entangled would this result in monism, and even then, entanglement—in the form of ontological holism—would be a genuine feature of our world.

Bell (1997) claimed that non-local correlations "cry out for explanation." By saying what's special about entangled physical systems, the present account goes further in answering this demand. 
Of course, if one adopts an interpretation which features a universal wavefunction with widespread entanglement, then we may be drawn to a version of monism on this view as well. However, there still may be a level of description at which it's appropriate to deploy effective wavefunctions which will be more restrictive in ascribing non-separable quantum states. If so, this approach may be deployed at that level: (all and only) the effectively entangled particles ontologically depend on the wholes they compose. This sort of non-global and effective application of the common ground explanation is unavailable on wavefunction realism, which is a global claim about fundamental reality.

The non-global common ground explanation of non-locality sketched here is incomplete in several respects. Most significantly, substantial work is required to fully integrate it into the extant interpretations of quantum theory. That said, it is prima facie open to any interpretation that posits systems of particles in non-separable quantum states to regard particles in such systems as ontologically dependent on the wholes they compose. The details of how such entangled states come about and are dissolved can be treated as a separate matter.

Thus, providing a common ground explanation of non-locality doesn't require wavefunction realism. One can instead retain entanglement as a genuine feature of our world by recognizing part-on-whole dependence. This alternative does a better job of explaining what's special about systems that display non-local behavior.

\section{Implications for spacetime fundamentality}

Our primary aim in the foregoing discussion has been to recommend entanglement realism over views that we see as eliminative. The discussion also has some important implications for the role of spacetime in our thinking about quantum theory.

A common feature of both of the approaches criticized above---Super-Humeanism and wavefunction realism---is the fundamental place they give to spatialtemporal (or quasi-spatialtemporal) relations. First, consider the Super-Humean. Esfeld and Deckert maintain that the only features of matter points are their spatiotemporal relations. This elevates background spacetime to an almost analytic status; the view cannot be articulated in a world in which fundamental spacetime or spacetime relations cannot be defined. Second, consider 
wavefunction realism. On this view, ordinary 3-dimensional space has been replaced by a fundamental $3 n$-dimensional configuration space. But, the analogous "quasi-spatial" relations between points in configuration space are given fundamental status. Thus, both views eschew putative entanglement relations in favor of an ontology of only (quasi-)spatiotemporal relations. However, this simplified ontology has certain costs. We've already discussed the potential explanatory cost in eliminating entanglement, but there is another important cost as well: the commitment to only (quasi-)spatiotemporal relations leaves hostages to fortune.

In the case of wavefunction realism, it is an open question whether the approach can be extended beyond ordinary non-relativistic quantum mechanics (Wallace and Timpson 2010; although see Ney forthcoming for a response). In the case of Super-Humeanism, the need to adopt something like a "primitive ontology" interpretation of quantum mechanics both leaves hostages to relativistic fortune and offends against the naturalistic metaphysical spirit by allowing background metaphysical preconceptions to dictate the choice of an idiosyncratic approach to a physical theory.

Moreover, if spacetime is emergent as some approaches to quantum gravity seem to suggest (Huggett and Wüthrich 2013), then clearly approaches that seek to eliminate relations of entanglement in favor of fundamental (quasi-)spatiotemporal relations will be untenable. This again recommends a common sense realism about entanglement relations over eliminativism.

\section{References}

Albert, D. Z. (1996). "Elementary quantum metaphysics" in Cushing, J., Fine, A, and Goldstein, S. eds., Bohmian mechanics and quantum theory: An appraisal, pp. 277-284. Springer.

Albert, D. Z. (2013). "Wave function realism" in Ney, A., and Albert, D. eds., The wave function: Essays on the metaphysics of quantum mechanics, pp. 52-57, Oxford University Press.

Barrett, J. A. (1999). The quantum mechanics of minds and worlds. Oxford University Press.

Bell, J. S. (1997). "Indeterminism and nonlocality" in Driessen, A., and Suarez, A. eds., Mathematical Undecidability, Quantum Nonlocality and the Question of the Existence of God, pp. 83-100, Springer.

Bell, J. S. (2004). Speakable and unspeakable in quantum mechanics: Collected papers on quantum philosophy. Cambridge university press.

Bhogal, H. and Perry, Z. (2017). "What the humean should say about entanglement." Noûs, 51(1):74-94.

Callender, C. (2015). "One world, one beable." Synthese, 192(10):3153-3177.

Esfeld, M. (2004). "Quantum entanglement and a metaphysics of relations." Studies in History 
and Philosophy of Science Part B: Studies in History and Philosophy of Modern Physics, 35(4):601-617.

Esfeld, M. (2014). "Quantum humeanism, or: Physicalism without properties.” The Philosophical Quarterly, 64(256):453-470.

Esfeld, M. and Deckert, D.-A. (2017). A minimalist ontology of the natural world. Routledge.

Fuchs, C. A., Mermin, N. D., and Schack, R. (2014). "An introduction to QBism with an application to the locality of quantum mechanics." American Journal of Physics, 82(8):749-754.

Healey, R. (2012). "Quantum theory: A pragmatist approach." The British Journal for the Philosophy of Science, 63(4):729-771.

Healey, R. (2016) "Holism and Nonseparability in Physics" in Zalta, E. N. ed., The Stanford Encyclopedia of Philosophy. Metaphysics Research Lab, Stanford.

Healey, R. (2017). "Quantum-bayesian and pragmatist views of quantum theory" in Zalta, E. N. ed., The Stanford Encyclopedia of Philosophy. Metaphysics Research Lab, Stanford University. Huggett, N. and Wüthrich, C. (2013) "Emergent spacetime and empirical (in)coherence." Studies in History and Philosophy of Science Part B: Studies in History and Philosophy of Modern Physics.

Ismael, J. (forthcoming). "Space, Quantum Mechanics, and Bohm's Fish Tank" in Glick, D., Darby, G., and Marmodoro, A., eds., The foundation of reality: Space, time, and fundamentality. Oxford University Press.

Ismael, J. and Schaffer, J. (2016). "Quantum holism: Nonseparability as common ground." Synthese, pp. 1-30.

Koslicki, K. (2012). "Varieties of ontological dependence" in Correia, F. and Schnieder, B. eds., Metaphysical grounding: Understanding the structure of reality, pp. 186--213, Cambridge University Press.

Lewis, D. (1986). Philosophical Papers, volume II. Oxford University Press.

Lewis, D. (2009). "Ramseyan humility" in Braddon-Mitchell, D. and Nola, R., eds., Conceptual Analysis and Philosophical Naturalism, pp. 203-222, MIT Press.

Loewer, B. (1996). "Humean supervenience." Philosophical Topics, 24(1):101-127.

Miller, E. (2014). "Quantum entanglement, Bohmian mechanics, and Humean supervenience." Australasian Journal of Philosophy, 92(3):567-583.

Ney, A. (2012). "The status of our ordinary three dimensions in a quantum universe." Noûs, 46(3):525-560.

Ney, A. (2013). "Ontological reduction and the wave function ontology" in Ney, A. and Albert. D.Z. eds., The wave function: Essays on the metaphysics of quantum mechanics, pp.168--183, Oxford University Press.

Ney, A. (2015). "Fundamental physical ontologies and the constraint of empirical coherence: a defense of wave function realism." Synthese, 192(10):3105-3124.

Ney, A. (forthcoming). "Wave function realism in a relativistic setting" in Glick, D., Darby, G. and Marmodoro, A., editors, The foundation of reality: Space, time, and fundamentality. Oxford University Press.

Norsen, T. (2016). "Quantum solipsism and non-locality." Quantum Nonlocality and Reality, 50, 204-237.

North, J. (2013). "The structure of a quantum world" in Ney, A. and Albert. D.Z. eds., The wave function: Essays on the metaphysics of quantum mechanics, pp 184-202, Oxford University Press.

Maudlin, T. (2011). Quantum non-locality and relativity: Metaphysical intimations of modern physics. John Wiley \& Sons.

Reichenbach, H. (1971). The direction of time. University of California Press. 
Schaffer, J. (2010). "Monism: The priority of the whole." Philosophical Review, 119.1 (2010): 31-76.

Schaffer, J. (2017). "Laws for metaphysical explanation." Philosophical Issues, 27(1), 302-321.

Schrödinger, E. (1935). "Discussion of probability relations between separated systems." Mathematical Proceedings of the Cambridge Philosophical Society, Vol. 31, No. 4, pp. 555-563.

Teller, P. (1986). "Relational holism and quantum mechanics." The British Journal for the Philosophy of Science, 37(1), 71-81.

Wallace, D., \& Timpson, C. G. (2010). "Quantum mechanics on spacetime I: Spacetime state realism." The British Journal for the Philosophy of Science, 61(4), 697-727.

Wallace, D. (2012). The emergent multiverse: Quantum theory according to the Everett interpretation. Oxford University Press. 